Review

\title{
Polynomial Approximation on Unbounded Subsets, Markov Moment Problem and Other Applications
}

\section{Octav Olteanu}

Department of Mathematics-Informatics, University Politehnica of Bucharest, 060042 Bucharest, Romania; octav.olteanu@upb.ro or octav.olteanu50@gmail.com

Received: 1 September 2020; Accepted: 23 September 2020; Published: 25 September 2020

\begin{abstract}
This paper starts by recalling the author's results on polynomial approximation over a Cartesian product $A$ of closed unbounded intervals and its applications to solving Markov moment problems. Under natural assumptions, the existence and uniqueness of the solution are deduced. The characterization of the existence of the solution is formulated by two inequalities, one of which involves only quadratic forms. This is the first aim of this work. Characterizing the positivity of a bounded linear operator only by means of quadratic forms is the second aim. From the latter point of view, one solves completely the difficulty arising from the fact that there exist nonnegative polynomials on $\mathbb{R}^{n}, n \geq 2$, which are not sums of squares.
\end{abstract}

Keywords: polynomial approximation; Hahn-Banach theorem; Markov moment problem; determinacy; quadratic forms

\section{Introduction}

The present paper refers mainly to various aspects of the classical multidimensional Markov moment problem and its relationship with polynomial approximation on special closed unbounded subsets $A$ of $\mathbb{R}^{n}$, namely on Cartesian products of closed unbounded intervals. We shall use the notations:

$$
\mathbb{N}=\{0,1,2, \ldots\}, \mathbb{R}_{+}=[0, \infty), \varphi_{j}(t)=t^{j}=t_{1}^{j_{1}} \ldots t_{n}^{j_{n}}, t=\left(t_{1}, \ldots, t_{n}\right) \in A \sqsubseteq \mathbb{R}^{n}, j=\left(j_{1}, \ldots, j_{n}\right) \in \mathbb{N}^{n}
$$

The cases $A=\mathbb{R}^{n}$ and, respectively, $A=\mathbb{R}_{+}^{n}, n \geq 1$ will be under attention, since they are the most important. The case $n=1$ will follow as a consequence, the explicit form of nonnegative polynomials over $\mathbb{R}$ and, respectively, on $\mathbb{R}_{+}$being well known (see [1]). The classical real moment problem can be formulated as follows: find necessary and sufficient conditions on a sequence $\left(y_{j}\right)_{j \in \mathbb{N} n}$ of real numbers, for the existence of a positive linear functional $T \in L_{+}(E, \mathbb{R})$, such that

$$
T\left(\varphi_{j}\right)=y_{j}, j \in \mathbb{N}^{n} .
$$

The numbers $y_{j}, j \in \mathbb{N}^{n}$ are called the moments with respect to the solution $T$. Here $E$ is a Banach function space containing both polynomials as well as continuous compactly supported real-valued functions on $A$. In particular, it results that $T$ can be represented by a positive regular Borel measure on $A$. In case of a Markov moment problem, one requires also an upper constraint on $T$ such as

$$
T \leq P \text { on } E,
$$

where $P$ is a continuous convex functional on $E$. Alternately, a condition of the following type could be imposed

$$
T_{1}(x) \leq T(x) \leq T_{2}(x), x \in E_{+}
$$


where $T_{1}, T_{2}$ are two given continuous linear functionals on $E$. Continuity of $P, T_{1}, T_{2}$ lead to continuity of the solution $T$ of the moment interpolation problem (1) satisfying (2) (respectively, (3)) and, in addition, controls the norm of the solution $T$. In the sequel, we restrict ourselves to the case when $E=L_{\mu}^{1}(A)$, $\mu$ being a positive regular Borel moment determinate measure on $A$. Consider the Markov moment problem of characterizing existence (and eventually uniqueness) of a linear form $T: L_{\mu}^{1}(A) \rightarrow \mathbb{R}$ which satisfies (3), with $T_{1}=\mathbf{0}$ and $T_{2}$ being a given (positive) continuous linear form on $L_{\mu}^{1}(A)$ ). The first step is to define the unique linear form $T_{0}$ on the subspace $\mathcal{P}$ of polynomials such that (1) is verified

$$
T_{0}: \mathcal{P} \rightarrow \mathbb{R}, T_{0}\left(\sum_{j \in J_{0}} \alpha_{j} \varphi_{j}\right)=\sum_{j \in J_{0}} \alpha_{j} y_{j}
$$

where $J_{0} \subset \mathbb{N}^{n}$ is an arbitrary finite subset and the coefficients $\alpha_{j}, j \in J_{0}$ are arbitrary real numbers. Extending $T_{0}$ to a linear functional $T$ on $L_{\mu}^{1}(A)$ such that

$$
0 \leq T(\varphi) \leq T_{2}(\varphi), \forall \varphi \in\left(L_{\mu}^{1}(A)\right)_{+}
$$

is described in Sections 2 and 3 below. The continuity of $T$ and density of $\mathcal{P}$ in $L_{\mu}^{1}(A)$ lead to the uniqueness of the solution $T$. This method works only for moment determinate measures $\mu$. Recall that a measure $\mu$ is called moment determinate ( $M$-determinate) if it is uniquely determinate by its moments

$$
m_{j}=m_{j}(\mu)=\int_{A} t^{j} d \mu, j \in \mathbb{N}^{n} .
$$

The first aim of this review paper is to characterize the existence (and uniqueness) of the solution $T$ for the Markov moment problem ((1) and (4)), in terms of quadratic forms. This aim is partially achieved in Section 3. Secondly, positivity of a bounded linear operator is completely characterized only by means of quadratic forms. This goal is completely attained. The interested reader can find more information on the moment problem in the monographs [1-4]. Basic results in measure theory, Banach lattices, order complete Banach lattices, related examples and inequalities, and new aspects of the Hahn-Banach theorem can be found in [5-9]. This paper is directly related to the references [4,10-16] and indirectly linked with the works $[5-9,17-38]$. The interested reader can find connections of the moment problem with other fields of analysis (other than polynomial approximation), such as operator theory, fixed point theory, optimization, and inverse problems. At the end of the Introduction, it is worth noticing that all our results are valid not only for real valued linear (respectively convex continuous) functionals, but also for operators having as codomain an order complete Banach lattice $F$. The order completeness assumption allows applying the Hahn-Banach result stated in Section 2 (Theorem 1 below). Other recent results on the Hahn-Banach theorem, sandwich theorem and/or their applications have been published in [39-42].

The rest of the paper is organized as follows. Section 2 reviews the methods used along this work, specifying some of the corresponding references and recalling a well-known extension result for linear positive operators, which holds preserving linearity and positivity. In Section 3, the main results specified in the Abstract are stated and partially proved. Section 4 concludes the paper.

\section{Materials and Methods}

The basic methods applied along this work are:

(1) Applying the next result, Theorem 1 stated below, sometimes called Lemma of the majorizing subspace (see $[3-5,11-16,21,22,24]$ for the proof or/and related applications), accompanied by polynomial approximation, to prove the existence of a positive solution $T$. Let $E_{1}$ be an ordered 
vector spaces for which the positive cone $E_{1,+}$ is generating $\left(E_{1}=E_{1,+}-E_{1,+}\right)$. Recall that in such an ordered vector space $E_{1}$, a vector subspace $S$ is called a majorizing subspace if

for any $x \in E_{1}$, there exists $s \in S$ such that $x \leq s$.

Theorem 1. Let $E_{1}$ be an ordered vector space whose positive cone is generating, $S \subset E_{1}$ a majorizing vector subspace, $F$ an order complete vector space, $T_{0}: S \rightarrow F$ a positive linear operator. Then $T_{0}$ admits a linear positive extension $T: E_{1} \rightarrow F$.

Using other variants of the Hahn-Banach theorem (separation theorem). For more general Hahn-Banach type theorems see [17-20].

Characterizing the existence of the solution $T$ in terms of its moments $y_{j}, j \in \mathbb{N}^{n}$.

(2) Measure theory detailed results discussed in [7].

(3) Polynomial approximation on unbounded subsets, (see [4,10-16]) recalled and applied in Section 3. Characterizing the existence of the solution $T$ in terms of its moments $y_{j}, j \in \mathbb{N}^{n}$ and quadratic forms. Uniqueness of the solution follows too. Applying the notion of a moment-determinate measure. Establishing determinacy or indeterminacy of a measure requires special criterions $($ see $[3,29,30])$.

(4) Evaluating the norm of the solution $T$ in terms of the norm of the given continuous linear functional (or operator) $T_{2}$, under conditions (4). This goal is achieved in theorems of Section 3 as well.

\section{Results}

\subsection{Solving Markov Moment Problem Over Unbounded Subsets via Polynomial Approximation}

Polynomial approximation recalled below allow proving the existence as well as the uniqueness of the solution of some classical moment problems on unbounded subsets.

Lemma 1. Let $\psi:[0, \infty) \rightarrow R_{+}$be a continuous function, such that $\lim _{t \rightarrow \infty} \psi(t) \in R_{+}$exists. Then there is a decreasing sequence $\left(h_{l}\right)_{l}$ in the linear hull of the functions

$$
\varphi_{k}(t)=\exp (-k t), k \in \mathbb{N}, t \in[0, \infty),
$$

such that $h_{l}(t)>\psi(t), t \geq 0, l \in \mathrm{N}, \lim h_{l}=\psi$ uniformly on $[0, \infty)$. There exists a sequence of polynomial functions $\left(\widetilde{p}_{l}\right)_{l \in \mathbb{N}}, \widetilde{p}_{l} \geq h_{l}>\psi, \lim \widetilde{p}_{l}=\psi$, uniformly on compact subsets of $[0, \infty)$.

Lemma 2. Let $v$ be an M-determinate positive regular measure on $[0, \infty)$, with finite moments of all natural orders. If $\psi,\left(\widetilde{p}_{l}\right)_{l}$ are as in Lemma 1 , then there exists a subsequence $\left(\widetilde{p}_{l m}\right)_{m}$, such that $\widetilde{p}_{l_{m}} \rightarrow \psi$ in $L_{v}^{1}([0 . \infty))$ and uniformly on compact subsets. In particular, it follows that the positive cone $\mathcal{P}_{+}$of nonnegative polynomials is dense in the positive cone $\left(L_{v}^{1}([0 . \infty))\right)_{+}$of $L_{v}^{1}([0 . \infty))$.

Lemma 3. Let $A \subset R^{n}$ be an unbounded closed subset, and $v$ an $M$-determinate positive regular Borel measure on $A$, with finite moments of all natural orders. Then for any $x \in C_{0}(A), x(t) \geq 0, \forall t \in A$, there exists a sequence $\left(p_{m}\right)_{m}, p_{m} \in P_{+}, p_{m} \geq x, p_{m} \rightarrow x$ in $L_{v}^{1}(A)$. In particular, we have

$$
\lim \int_{A} p_{m}(t) d v=\int_{A} x(t) d v
$$

$\mathcal{P}_{+}$is dense in $\left(L_{v}^{1}(A)\right)_{+}$, and $\mathcal{P}$ is dense in $L_{v}^{1}(A)$. (Here $C_{0}(A)$ is the vector space of all continuous compactly supported real valued functions defined on $A)$. 
Proof. Let consider the sublattice $X \subset L_{v}^{1}(A)$ of all function $\psi$ such that $|\psi|$ is dominated by some polynomial $p$ on $A$. To prove the assertions of the statement, it is sufficient to show that for any $x \in\left(C_{0}(A)\right)_{+}$, we have

$$
Q_{1}(x)=\inf \left\{\int_{A} p(t) d v, p \geq x, p \in \mathcal{P}\right\}=\int_{A} x(t) d v .
$$

Obviously, one has

$$
Q_{1}(x) \geq \int_{A} x(t) d v
$$

To prove the converse, we define the linear form

$$
T_{0}: X_{0}=\mathcal{P} \oplus S p\{x\} \rightarrow \mathbb{R}, T_{0}(p+\alpha x)=\int_{A} p(t) d v+\alpha Q_{1}(x), p \in \mathcal{P}, \alpha \in \mathbb{R} .
$$

Next, we show that $T_{0}$ is positive on $X_{0}$. In fact, for $a<0$, one has (from the definition of $Q_{1}$, which is a sublinear functional on $X$ )

$$
p+\alpha x \geq 0 \Rightarrow p \geq-a x \Rightarrow(-a) Q_{1}(x)=Q_{1}(-a x) \leq \int_{A} p(t) d v \Rightarrow T_{0}(p+a x) \geq 0 .
$$

If $a \geq 0$, we infer that

$$
\begin{gathered}
0=Q_{1}(\mathbf{0})=Q_{1}(a x+(-a x)) \leq a Q_{1}(x)+Q_{1}(-a x) \\
\Rightarrow \int_{A} p(t) d v \geq Q_{1}(-a x) \geq-a Q_{1}(x) \Rightarrow T_{0}(p+a x) \geq 0 .
\end{gathered}
$$

Whence, in both possible cases, we have $x_{0} \in\left(X_{0}\right)_{+} \Rightarrow T_{0}\left(x_{0}\right) \geq 0$. Since $X_{0}$ contains the space of polynomials functions, which is a majorizing subspace of $X$, according to Theorem 1 , there exists a linear positive extension $T: X \rightarrow \mathbb{R}$ of $T_{0}$, which is continuous on $C_{0}(A)$, with respect to the sup-norm. Therefore, $T$ has a representation by means of a positive Borel regular measure $\mu$ on $A$, such that

$$
T(x)=\int_{A} x(t) d \mu, x \in C_{0}(A) .
$$

Let $p \in \mathcal{P}_{+}$be a nonnegative polynomial function. There is a nondecreasing sequence $\left(x_{m}\right)_{m}$ of continuous nonnegative function with compact support, such that $x_{m} \uparrow p$, pointwise on $A$. Positivity of $T$ and Lebesgue dominated convergence theorem for $\mu$ yield

$$
\int_{A} p d v=T(p) \geq \sup _{m} T\left(x_{m}\right)=\sup _{m} \int_{A} x_{m}(t) d \mu=\int_{A} p d \mu, p \in \mathcal{P}_{+} .
$$

Thanks to Haviland theorem (see [21]), there exists a positive Borel regular measure $\lambda$ on $A$, such that

$$
\lambda(p)=v(p)-\mu(p) \Leftrightarrow v(p)=\lambda(p)+\mu(p), p \in P
$$

Since $v$ is assumed to be $M$-determinate, it follows that

$$
v(B)=\mu(B)+\lambda(B),
$$

for any Borel subset $B$ of $A$. From this last assertion, approximating each $x \in\left(L_{v}^{1}(A)\right)$, by a nondecreasing sequence of nonnegative simple functions, and also using the Lebesgue convergence theorem, one obtains firstly for positive functions, then for arbitrary $v$-integrable functions $x$ :

$$
\int_{A} x d v=\int_{A} x d \mu+\int_{A} x d \lambda, \quad x \in L_{v}^{1}(A)
$$


In particular, we must have

$$
\int_{A} x d v \geq T(x)=T_{0}(x)=Q_{1}(x), x \in\left(C_{0}(A)\right)_{+} .
$$

Now (5) and (6) conclude the proof.

Using the Bernstein polynomial approximation theorem in several variables and Lemma 3 for $n=1$, one can prove the following result (see $[13,15])$.

Lemma 4. Let $v=v_{1} \times \ldots \times v_{n}$ be a product of $n M$-determinate positive regular Borel measures on $\mathbb{R}_{+}=[0, \infty)$, with finite moments of all natural orders. Then we can approximate any nonnegative continuous compactly supported function in $X=L_{v}^{1}\left(\mathbb{R}_{+}^{n}\right)$ by means of sums of tensor products $p_{1} \otimes \ldots \otimes p_{n}, p_{j}$ positive polynomial on the real nonnegative semiaxis, in variable

$$
t_{j} \in[0, \infty), j=1, \ldots, n \text {, where }\left(p_{1} \otimes \ldots \otimes p_{n}\right)\left(t_{1}, \ldots, t_{n}\right)=p_{1}\left(t_{1}\right) \ldots p_{n}\left(t_{n}\right) .
$$

Notice that a similar result holds for products of $M$-determinate positive regular measures on $\mathbb{R}$, with finite moments of all natural orders.

The following statement holds for any closed unbounded subset $A \subset \mathbb{R}^{n}$, hence does not depend on the form of positive polynomials on $A$. As usual we denote by

$$
\varphi_{j}(t)=t_{1}^{j_{1}} \ldots t_{n}^{j_{n}}, j=\left(j_{1}, \ldots, j_{n}\right) \in \mathbb{N}^{n}, t=\left(t_{1}, \ldots, t_{n}\right) \in A .
$$

Theorem 2. Let $A$ be a closed unbounded subset of $\mathbb{R}^{n}, Y$ an order complete Banach lattice, $\left(y_{j}\right)_{j \in \mathbb{N}^{n}}$ a given sequence in $Y, v$ a positive regular $M$ - determinate Borel measure on $A$, with finite moments of all orders. Let $T_{2} \in B\left(L_{v}^{1}(A), Y\right)$ be a linear positive bounded operator from $L_{v}^{1}(A)$ to $Y$. The following statements are equivalent

(a) there exists a unique linear operator $\widetilde{T} \in B\left(L_{v}^{1}(A), Y\right)$ such that $\widetilde{T}\left(\varphi_{j}\right)=y_{j}, j \in \mathbb{N}^{n}, \widetilde{T}$ is between 0 and $T_{2}$ on the positive cone of $L_{v}^{1}(A)$, and $\|\widetilde{T}\| \leq\left\|T_{2}\right\|$;

(b) for any finite subset $J_{0} \subset \mathbb{N}^{n}$, and any $\left\{a_{j}\right\}_{j \in J_{0}} \subset \mathbb{R}$, we have

$$
\sum_{j \in J_{0}} a_{j} \varphi_{j} \geq 0 \text { on } A \Rightarrow 0 \leq \sum_{j \in J_{0}} a_{j} y_{j} \leq \sum_{j \in J_{0}} a_{j} T_{2}\left(\varphi_{j}\right)
$$

Proof. Observe that the assertion (b) says that

$$
0 \leq T_{0}(p) \leq T_{2}(p), p \in \mathcal{P}_{+},
$$

where $T_{0}: \mathcal{P} \rightarrow Y$ is the unique linear operator which verifies the interpolation conditions (1). Thus $(a) \Rightarrow(b)$ is obvious. To prove the converse, consider the vector subspace $E_{1} \subset L_{v}^{1}(A)$ of all functions $\varphi \in L_{v}^{1}(A)$ verifying

$$
|\varphi(t)| \leq p(t) \quad \forall t \in A
$$

for some polynomial $p$. Clearly, $E_{1}$ contains the subspace of polynomials as well as the subspace of continuous compactly supported real valued functions. On the other hand, the subspace of polynomials is a majorizing subspace in $E_{1}$ and according to the first inequality (7), $T_{0}$ in positive as a linear operator on $\mathcal{P}$. Application of Theorem 1 yields the existence of a positive linear extension $T: E_{1} \rightarrow Y$ of 
$T_{0}$. Let $x$ be a nonnegative continuous compactly supported function on $A$ and $\left(p_{m}\right)_{m}$ a sequence of polynomials with the properties specified in Lemma 3. According to the second inequality (7), we have

$$
T\left(p_{m}\right)=T_{0}\left(p_{m}\right) \leq T_{2}\left(p_{m}\right) \text { for all } m \in \mathbb{N} .
$$

Our next goal is to prove that

$$
T(x) \leq T_{2}(x)
$$

Assuming the contrary, we should have $T_{2}(x)-T(x) \notin Y_{+}$. Since $Y_{+}$is closed, a Hahn-Banach separation theorem leads to the existence of a positive linear form $y^{*}$ in the dual $Y^{*}$ of $Y$, verifying

$$
y^{*}\left(T_{2}(x)-T(x)\right)<0
$$

The positive linear form $y^{*} \circ T$ has a representing positive regular Borel measure $\mu$, which for Fatou lemma can be applied; we infer that

$$
y^{*}(T(x)) \leq \liminf f_{m} y^{*}\left(T\left(p_{m}\right)\right) \leq \liminf f_{m} y^{*}\left(T_{2}\left(p_{m}\right)\right)=y^{*}\left(T_{2}\left(\lim _{m} p_{m}\right)\right)=y^{*}\left(T_{2}(x)\right) .
$$

Relations (9) and (10) lead to

$$
y^{*}\left(T_{2}(x)\right)<y^{*}(T(x)) \leq y^{*}\left(T_{2}(x)\right)
$$

which imply the contradiction $y^{*}\left(T_{2}(x)\right)<y^{*}\left(T_{2}(x)\right)$. Hence the assumption $T_{2}(x)-T(x) \notin Y_{+}$was false so that we must have $T_{2}(x)-T(x) \in Y_{+}$, i.e., (8) is proved. Now let $\varphi \in C_{0}(A)$ be arbitrary. According to the preceding considerations, we obtain

$$
|T(\varphi)|=\left|T\left(\varphi^{+}\right)-T\left(\varphi^{-}\right)\right| \leq T\left(\varphi^{+}\right)+T\left(\varphi^{-}\right) \leq T_{2}\left(\varphi^{+}\right)+T_{2}\left(\varphi^{-}\right)=T_{2}(|\varphi|) .
$$

Since the norm on $Y$ is solid $\left(\left|y_{1}\right| \leq\left|y_{2}\right| \Rightarrow\left\|y_{1}\right\| \leq\left\|y_{2}\right\|\right)$, we infer that

$$
\|T(\varphi)\| \leq\left\|T_{2}(|\varphi|)\right\| \leq\left\|T_{2}\right\|\||\varphi|\|_{1}=\left\|T_{2}\right\|\|\varphi\|_{1}, \quad \forall \varphi \in C_{0}(A) .
$$

Using the fact that $C_{0}(A)$ is dense in $L_{v}^{1}(A)$ (see [7]), the last evaluation leads to the existence of a linear extension $\widetilde{T}: L_{v}^{1}(A) \rightarrow Y$ of $T$, such that

$$
\|\widetilde{T}(\varphi)\| \leq\left\|T_{2}\right\| \varphi\left\|_{1}, \quad\right\| \varphi \|_{1}=\int_{A}|\varphi(t)| d v, \quad \varphi \in L_{v}^{1}(A) .
$$

It results $\|\widetilde{T}\| \leq\left\|T_{2}\right\|$ and the positivity of $\widetilde{T}$ is a consequence of the positivity of $T$, via continuity of the extension $\widetilde{T}$ and the density of $\left(C_{0}(A)\right)_{+}$in $\left(L_{v}^{1}(A)\right)_{+}$. We also notice that

$$
\widetilde{T}\left(\varphi_{j}\right)=T_{0}\left(\varphi_{j}\right)=y_{j}, j \in \mathbb{N}^{n}
$$

This concludes the proof.

We go on by recalling a result on the form of non-negative polynomials in a strip [23], which leads to a simple solution for the related Markov moment problem.

Theorem 3. Suppose that $p\left(t_{1}, t_{2}\right) \in \mathbb{R}\left[t_{1}, t_{2}\right]$ is non-negative on the strip $A=[0,1] \times \mathbb{R}$. Then $p\left(t_{1}, t_{2}\right)$ is expressible as

$$
p\left(t_{1}, t_{2}\right)=\sigma\left(t_{1}, t_{2}\right)+\tau\left(t_{1}, t_{2}\right) t_{1}\left(1-t_{1}\right) .
$$


Let $A=[0,1] \times \mathbb{R}, v$ a positive $M$ - determinate regular Borel measure on $A$, with finite moments of all orders, $X=L_{v}^{1}(A), \varphi_{j}\left(t_{1}, t_{2}\right)=t_{1}^{j_{1}} t_{2}^{j_{2}}, j=\left(j_{1}, j_{2}\right) \in \mathbb{N}^{2},\left(t_{1}, t_{2}\right) \in A$. Let $Y$ be on order complete Banach lattice, $\left(y_{j}\right)_{j \in \mathbb{N}^{2}}$ a sequence of given elements in $Y$.

Theorem 4. Let $F_{2} \in B_{+}(X, Y)$ be a linear bounded positive operator from $X$ to $Y$. The following statements are equivalent

(a) there exists a unique bounded linear operator $F: X \rightarrow Y$, such that $F\left(\phi_{j}\right)=y_{j}, \forall j \in N^{2}, F$ is between zero and $F_{2}$ on the positive cone of $X,\|F\| \leq\left\|F_{2}\right\| ;$

(b) for any finite subset $J_{0} \subset \mathbb{N}^{2}$, and any $\left\{\lambda_{j} ; j \in J_{0}\right\} \subset \mathbb{R}$, we have

$$
\begin{gathered}
0 \leq \sum_{i, j \in J_{0}} \lambda_{i} \lambda_{j} y_{i+j} \leq \sum_{i, j \in J_{0}} \lambda_{i} \lambda_{j} F_{2}\left(\varphi_{i+j}\right) ; \\
0 \leq \sum_{i, j \in J_{0}} \lambda_{i} \lambda_{j}\left(y_{i_{1}+j_{1}+1, i_{2}+j_{2}}-y_{i_{1}+j_{1}+2, i_{2}+j_{2}}\right) \leq \\
\sum_{i, j \in J_{0}} \lambda_{i} \lambda_{j}\left(F_{2}\left(\varphi_{i_{1}+j_{1}+1, i_{2}+j_{2}}-\varphi_{i_{1}+j_{1}+2, i_{2}+j_{2}}\right)\right), i=\left(i_{1}, i_{2}\right), j=\left(j_{1}, j_{2}\right) \in J_{0}
\end{gathered}
$$

The proof follows from Theorem 3 and Theorem 2.

Theorem 5. Let $X$ be as in Lemma $4,\left(y_{j}\right)_{j \in \mathbb{N}^{n}}$ a sequence in $Y$, where $Y$ is an order complete Banach lattice, $F_{2} \in B_{+}(X, Y)$. The following statements are equivalent

(a) there exists a unique (bounded) linear operator $F \in B(X, Y)$ such that, $F\left(\varphi_{j}\right)=y_{j}, j \in \mathbb{N}^{n}, F$ is between zero and $F_{2}$ on the positive cone of $X,\|F\| \leq\left\|F_{2}\right\| ;$

(b) for any finite subset $J_{0} \subset \mathrm{N}^{n}$ and any $\left\{\lambda_{j} ; j \in J_{0}\right\} \subset \mathrm{R}$, we have

$$
\begin{gathered}
\sum_{j \in J_{0}} \lambda_{j} \phi_{j}(t) \geq 0 \forall t \in \mathrm{R}_{+}^{n} \Rightarrow \sum_{j \in J_{0}} \lambda_{j} y_{j} \in Y_{+} ; \text {for any finite subsets } J_{k} \subset \mathrm{N}, k=1, \ldots, n, \\
\text { and any }\left\{\lambda_{j_{k}}\right\}_{j_{k} \in J_{k}} \subset R, k=1, \ldots, n \text {, the following relations hold } \\
\sum_{i_{1}, j_{1} \in J_{1}}\left(\ldots\left(\sum_{i_{n}, j_{n} \in J_{n}} \lambda_{i_{1}} \lambda_{j_{1}} \ldots \lambda_{i_{n}, j_{n} \in J_{n}} \lambda_{i_{1}} \lambda_{j_{n}} \lambda_{j_{1}} \ldots \lambda_{i_{n}} \lambda_{j_{n}} y_{i_{1}+j_{1}+l_{1}, \ldots, i_{n}+j_{n}+l_{n}}\right) \ldots\right) \leq
\end{gathered}
$$

Let $v=v_{1} \times \ldots \times v_{n}$ be a product of $n M-$ determinate positive regular Borel on $\mathbb{R}$, with finite moments of all natural orders. Let

$$
\varphi_{j}(t)=t_{1}^{j_{1}} \ldots t_{n}^{j_{n}}, j=\left(j_{1}, \ldots, j_{n}\right) \in \mathbb{N}^{n}, t=\left(t_{1}, \ldots, t_{n}\right) \in \mathbb{R}^{n}
$$

Obviously, a statement similar to that of Lemma 4 holds true when we replace $\mathbb{R}_{+}^{n}$ by $\mathbb{R}^{n}$. In the latter case, the polynomials $p_{j}, j=1, \ldots, n$ are nonnegative on the whole real axis, so that they are sums of squares. Applying such an approximation result and Theorem 1, one obtains the following theorem.

Theorem 6. Let $v$ be as above, $X=L_{v}^{1}\left(\mathbb{R}^{n}\right), Y$ an order complete Banach lattice, and $\left(y_{j}\right)_{j \in \mathbb{N}^{n}}$ a multi-indexed sequence in $Y$. Let $F_{2}: X \rightarrow Y$ be a positive linear bounded operator. The following statements are equivalent

(a) there exists a unique bounded linear operator $F: X \rightarrow Y$, such that $F\left(\phi_{j}\right)=y_{j}, \forall j \in \mathrm{N}^{n}, F$ is between zero and $F_{2}$ on the positive cone of $X,\|F\| \leq\left\|F_{2}\right\|$; 
(b) for any finite subset $J_{0} \subset \mathrm{N}^{n}$, and any $\left\{\lambda_{j} ; j \in J_{0}\right\} \subset \mathrm{R}$, we have

$$
\sum_{j \in J_{0}} \lambda_{j} \phi_{j}(t) \geq 0 \forall t \in \mathrm{R}^{n} \Rightarrow \sum_{j \in J_{0}} \lambda_{j} y_{j} \in Y_{+},
$$

for any finite subsets $J_{k} \subset \mathrm{N}, k=1, \ldots, n$, and any $\left\{\lambda_{j_{k}}\right\}_{j_{k} \in J_{k}} \subset R, k=1, \ldots, n$, the following relations hold

$$
\begin{aligned}
& \sum_{i_{1}, j_{1} \in J_{1}}\left(\ldots\left(\sum_{i_{n}, j_{n} \in J_{n}} \lambda_{i_{1}} \lambda_{j_{1}} \ldots \lambda_{i_{n}} \lambda_{j_{n}} y_{i_{1}+j_{1}, \ldots, i_{n}+j_{n}}\right) \ldots\right) \leq \\
& \sum_{i_{1}, j_{1} \in J_{1}}\left(\ldots\left(\sum_{i_{n}, j_{n} \in J_{n}} \lambda_{i_{1}} \lambda_{j_{1}} \ldots \lambda_{i_{n}} \lambda_{j_{n}} F_{2}\left(\varphi_{i_{1}+j_{1}, \ldots, i_{n}+j_{n}}\right) \ldots\right)\right.
\end{aligned}
$$

In what follows we solve an operator valued one dimensional classical Markov moment problem. Let $H$ be an arbitrary complex or real Hilbert space and $\mathcal{A}$ the real order vector space of all self-adjoint operators acting on $H$. The positive cone of $\mathcal{A}$ consists in all operators $U \in \mathcal{A}$, having the property: $\langle U h, h\rangle \geq 0 \forall h \in H$. Let $A \in \mathcal{A}$. Define $Y=Y(A)$ by

$$
Y_{1}=\{V \in \mathcal{A} ; V A=A V\}, Y=Y(A)=\left\{U \in Y_{1} ; U V=V U, \forall V \in Y_{1}\right\} .
$$

As is well-known, according to [5], $Y=Y(A)$ is an order complete Banach lattice. Let $X=C_{\mathbb{R}}(\sigma(A))$, where $\sigma(A) \subset[0, \infty)$ is the spectrum of the fixed positive self-adjoint operator $A$ acting on a complex (or real) Hilbert space $H$. Consider the space $Y(A)$ defined by (11). The following statement holds, with similar proof to that of the above result Theorem 5, thanks to the form of non-negative polynomials on $[0, \infty)$ (see [1]).

Proposition 1. Let $Y$ be defined by (11), $\left(U_{n}\right)_{n n \geq 0}$ be a sequence of operators in $Y$. The following statements are equivalent

(a) there exists a unique linear bounded operator $T: X \rightarrow Y$ such that the moment interpolation conditions $T\left(\varphi_{n}\right)=U_{n}, n \in \mathbb{N}$ are verified and $0 \leq T(\psi) \leq \psi(A), \forall \psi \in X_{+},\|T\| \leq 1 ;$

(b) for any finite subset $J_{0} \subset \mathbb{N}$ and any $\left\{\lambda_{j} ; j \in J_{0}\right\} \subset \mathbb{R}$, the following implication holds true

$$
\sum_{j \in J_{0}} \lambda_{j} t^{j} \geq 0, \forall t \in \sigma(A) \Rightarrow 0 \leq \sum_{j \in J_{0}} \lambda_{j} U_{j} \leq \sum_{j \in J_{0}} \lambda_{j} A^{j}
$$

(c) for any finite subset $J_{0} \subset \mathbb{N}$ and any $\left\{\lambda_{j} ; j \in J_{0}\right\} \subset \mathbb{R}$, the following relations hold

$$
0 \leq \sum_{i, j \in J_{0}} \lambda_{i} \lambda_{j} U_{i+j+k} \leq \sum_{i, j \in J_{0}} \lambda_{i} \lambda_{j} A^{i+j+k}, k \in\{0,1\}
$$

The proof of Proposition 1 is somehow similar to that of Theorem 2. However, in the former case uniform convergence of (nonnegative polynomials on $[0, \infty)$ ) on any compact subset ensured by Lemma 1 (or Lemma 2) is applied, while in the latter case polynomial approximation in $L^{1}$ norm, by nonnegative polynomials over the closed subset under attention is sufficient. When applying Lemma 1 , the involved compact subset of $[0, \infty)$ is the spectrum $\sigma(A)$.

Denote

$$
S_{1}^{+}(X)=\left\{T \in B_{+}(X) ; T\left(\bar{B}_{1, X}\right) \subseteq \bar{B}_{1, X}\right\}
$$


where $X=L_{v}^{1}(S)$, ( $S$ is a closed unbounded subset of $\mathbb{R}^{n}, v$ being $M$-determinate) and $\bar{B}_{1, X}$ is the closed unit ball in $X$, while $B_{+}(X)$ is the positive cone of the space $B(X)$ of all bounded linear operators from $X$ to itself. Let $V: \mathcal{P} \rightarrow X$ be a linear operator.

Corollary 1. The following statements are equivalent

(a) $\quad V$ has a positive linear extension $\widetilde{V} \in S_{1}^{+}(X)$;

(b) there exists $T \in S_{1}^{+}(X)$ such that

$$
0 \leq V(p) \leq T(p), \quad \forall p \in \mathcal{P}_{+} .
$$

Proof. Let $Y=X=L_{v}^{1}(S)$. Then $Y$ is an order complete Banach lattice. To prove $(b) \Rightarrow(a)$, one applies the corresponding implication from Theorem 2. Observe also that

$$
\|\widetilde{V}\| \leq 1 \text { if and only if } \widetilde{V}\left(\bar{B}_{1, X}\right) \subseteq \bar{B}_{1, X} .
$$

The converse implication is obvious. This concludes the proof.

Our next goal is to give some characterizations in terms of quadratic forms (when this fact is allowed by the form of positive polynomials by means of sums of squares).

Corollary 2. Let $X=L_{v}^{1}(\mathbb{R})$, where $v$ is a positive regular Borel $M$ - determinate measure on $\mathbb{R}$ (with finite moments of all orders), $x_{j}(t)=t^{j}, t \in \mathbb{R}, j \in \mathbb{N}$. Let $V: \mathcal{P} \rightarrow X$ be a linear operator. The following statements are equivalent

(a) V has a linear positive extension $\widetilde{V} \in S_{1}^{+}(X)$;

(b) there exists $T \in S_{1}^{+}(X)$ such that for any finite subset $\left\{\lambda_{j}\right\}_{j \in J_{0}} \subset \mathbb{R}$, the following relations hold

$$
0 \leq \sum_{i, j \in J_{0}} \lambda_{i} \lambda_{j} V\left(x_{i+j}\right) \leq \sum_{i, j \in J_{0}} \lambda_{i} \lambda_{j} T\left(x_{i+j}\right)
$$

\subsection{Characterizing Positivity of Some Bounded Linear Operators}

Let $v=v_{1} \times \ldots \times v_{n}$ be a product of $n M-$ determinate positive regular Borel on $\mathbb{R}$, with finite moments of all natural orders. Let

$$
\varphi_{j}(t)=t_{1}^{j_{1}} \ldots t_{n}^{j_{n}}, j=\left(j_{1}, \ldots, j_{n}\right) \in \mathbb{N}^{n}, t=\left(t_{1}, \ldots, t_{n}\right) \in \mathbb{R}^{n} .
$$

According to a similar statement to that of Lemma 4 stated above, each nonnegative continuous compactly supported function on $\mathbb{R}^{n}$ can be approximated by sums of polynomials of the form

$$
p_{1} \otimes \ldots \otimes p_{n}
$$

where $p_{k}$ is a nonnegative polynomial of variable $t_{k}$, over the entire real axis, $k=1, \ldots, n$. The next result is somehow converse to those of Section 3.1: now we assume that a continuous linear operator $T: X=L_{v}^{1}\left(\mathbb{R}^{n}\right) \rightarrow Y$ is given, where $Y$ is an arbitrary Banach space, endowed with a linear order relation that makes the positive cone $Y_{+}$to be closed (in particular, $Y$ can be an ordered Banach space). Then we characterize the positivity of $T$ in terms of quadratic forms only. The Hahn-Banach theorem plays no role here; only polynomial approximation is applied.

Theorem 7. Let $X, Y$ be as above and $T$ is a bounded linear operator from $X$ to $Y$. The following statements are equivalent 
(a) $T$ is nonnegative on the positive cone of $X$;.

(b) for any finite subsets

$$
J_{k} \subset \mathbb{N}, k=1, \ldots, n \text { and any }\left\{\lambda_{j_{k}}\right\}_{j_{k} \in J_{k}} \subset \mathbb{R}, k=1, \ldots, n,
$$

the following relation holds

$$
0 \leq \sum_{i_{1}, j_{1} \in J_{1}}\left(\ldots\left(\sum_{i_{n} \cdot j_{n} \in J_{n}} \lambda_{i_{1}} \lambda_{j_{1}} \ldots \lambda_{i_{n}} \lambda_{j_{n}} T\left(\varphi_{i_{1}+j_{1}}, \ldots, i_{n}+j_{n}\right)\right) \ldots\right) .
$$

Proof. Notice that $(b)$ says that $T$ is positive on the convex cone generated by special positive polynomials

$$
p_{1} \otimes \ldots \otimes p_{n}
$$

each factor of any term in the sum being nonnegative on the whole real axis. Consequently, $(a) \Rightarrow(b)$ is obvious. In order to prove the converse, observe that any nonnegative element of $X$ can be approximated by nonnegative continuous compactly supported functions. Such functions can be approximated by sums of tensor products of positive polynomials in each separate variable, the latter being sums of squares. The conclusion is that any nonnegative function from $X$ can be approximated in $X=L_{v}^{1}\left(\mathbb{R}^{n}\right)$ by sums of tensor products of squares of polynomials in each separate variable. However, on such special polynomials, $T$ admits nonnegative values, according to the condition (b). Now the desired conclusion is a consequence of the continuity of $T$, also using the fact that the positive cone of $Y$ is closed. This concludes the proof.

\section{Discussion}

The first aim was characterizing the existence and uniqueness of the solution for a class of Markov moment problem in terms of quadratic forms (see Section 3.1). This is only partially achieved (only one of the two inequalities appearing at point (b) referring to such characterizations involves signatures of quadratic forms). However, the aim of Section 3.2 (namely characterizing positivity of some bounded linear operators) is completely achieved by means of quadratic forms. Both subsections are based on polynomial approximation on special closed unbounded subsets. In Section 3.1, Hahn-Banach type results are applied as well. On the other hand, the proof of the polynomial approximation result of Lemma 3 is essentially using Theorem 1, which is a Hahn-Banach type result.

Funding: This research received no external funding.

Conflicts of Interest: The author declares no conflict of interest.

\section{References}

1. Akhiezer, N.I. The Classical Moment Problem and Some Related Questions in Analysis; Oliver and Boyd: Edinburgh-London, UK, 1965.

2. Krein, M.G.; Nudelman, A.A. Markov Moment Problem and Extremal Problems; American Mathematical Society: Providence, RI, USA, 1977.

3. Schmüdgen, K. The Moment Problem; In Graduate Texts in Mathematics; Springer: Berlin/Heidelberg, Germany; New York, NY, USA, 2017. [CrossRef]

4. Olteanu, O. Recent Results on Markov Moment Problem, Polynomial Approximation and Related Fields in Analysis; Generis Publishing: Chisinau, Republic of Moldova, 2020.

5. Cristescu, R. Ordered Vector Spaces and Linear Operators; Editura Academiei: Bucharest, Romania; Abacus Press: Tunbridge Wells, UK, 1976.

6. Niculescu, C.P.; Persson, L.-E. Convex functions and their applications. A contemporary approach. In CMS Books in Mathematics, 2nd ed.; Springer: New York, NY, USA, 2018; Volume 23. 
7. Rudin, W. Real and Complex Analysis, 3rd ed.; McGraw-Hill Book Company: London, UK, 1987.

8. Schaefer, H.H. Topological Vector Spaces, 3rd Printing Corrected; Springer: Berlin/Heidelberg, Germany; New York, NY, USA, 1971. [CrossRef]

9. Simons, S. From Hahn-Banach to Monotonicity, 2nd ed.; In Lecture Notes in Mathematics; Springer: New York, NY, USA, 2008.

10. Mihăilă, J.M.; Olteanu, O.; Udrişte, C. Markov-Type moment problems for arbitrary compact and some noncompact Borel subsets of $\mathbb{R}^{\mathrm{n}}$. Rev. Roum. Math Pures Appl. 2007, 52, 655-664.

11. Olteanu, O. Approximation and Markov moment problem on concrete spaces. Rend. Circ. Mat. Palermo 2014, 63, 161-172. [CrossRef]

12. Olteanu, O. Applications of Hahn-Banach principle to the moment problem. Poincare J. Anal. Appl. 2015, 1, 1-28. [CrossRef]

13. Olteanu, O. Markov moment problems and related approximation. Math. Rep. 2015, 17, 107-117.

14. Olteanu, O. Invariant subspaces and invariant balls of bounded linear operators. Bull. Math. Soc. Sci. Math. Roum. 2016, 59, 261-271.

15. Olteanu, O.; Mihăilă, J.M. Polynomial approximation on unbounded subsets and some applications. Asian J. Sci. Technol. 2018, 9, 8875-8890.

16. Olteanu, O.; Mihăilă, J.M. Markov moment problem in concrete spaces revisited. MathLAB J. 2020, 5, 82-91.

17. Olteanu, O. Convexité et prolongement d'opérateurs linéaires. C. R. Acad. Sci. Paris Série A 1978, 286, 511-514.

18. Olteanu, O. Théorèmes de prolongement d'opérateurs linéaires. Rev. Roum Math. Pures Appl. 1983, 28, 953-983.

19. Olteanu, O. Application de théorèmes de prolongement d'opérateurs linéaires au problème des moments e à une généralization d'un théorème de Mazur-Orlicz. C. R. Acad. Sci. Paris 1991, t. 313, Série I, 739-742.

20. Olteanu, O. From Hahn-Banach type theorems to the Markov moment problem, sandwich theorems and further applications. Mathematics 2020, 8, 1328. [CrossRef]

21. Haviland, E.K. On the momentum problem for distributions in more than one dimension. Am. J. Math. 1936, 58, 164-168. [CrossRef]

22. Kutateladze, S.S. Convex operators. Russ. Math. Surv. 1979, 34, 181-214. [CrossRef]

23. Marshall, M. Polynomials non-negative on a strip. Proc. Am. Math. Soc. 2010, 138, 1559-1567. [CrossRef]

24. Choquet, G. Le problème des moments. In Séminaire Choquet. Initiation à l'Analyse; Institut Henri Poincaré: Paris, France, 1962; Volume 1, 10p.

25. Berg, C.; Christensen, J.P.R.; Jensen, C.U. A remark on the multidimensional moment problem. Math. Ann. 1979, 243, 163-169. [CrossRef]

26. Berg, C.; Durán, A.J. The fixed point for a transformation of Hausdorff moment sequences and iteration of a rational function. Math. Scand. 2008, 103, 11-39. [CrossRef]

27. Cassier, G. Problèmes des moments sur un compact de $\mathbb{R}^{\mathrm{n}}$ et décomposition des polynomes à plusieurs variables. J. Funct. Anal. 1984, 58, 254-266. [CrossRef]

28. Gosse, L.; Runborg, O. Existence, uniqueness, and a constructive solution algorithm for a class of finite Markov moment problems. SIAM J. Appl. Math. 2008, 68, 1618-1640. [CrossRef]

29. Kleiber, C.; Stoyanov, J. Multivariate distributions and the moment problem. J. Multivar. Anal. 2013, 113, 7-18. [CrossRef]

30. Stoyanov, J.M.; Lin, G.D.; Kopanov, P. New checkable conditions for moment determinacy of probability distributions. SIAM Theory Probab. Appl. 2020, 65, 3. (in print). Originally published in the Russian journal Teoriya Veroyatnostei i ee Primeneniya 2020, 65, 634-648. [CrossRef]

31. Lemnete, L. An operator-valued moment problem. Proc. Am. Math. Soc. 1991, 112, 1023-1028. [CrossRef]

32. Lemnete-Ninulescu, L.; Zlătescu, A. Some new aspects of the L.-moment problem. Rev. Roum Math. Pures Appl. 2010, 55, 197-204.

33. Putinar, M. Positive polynomials on compact semi-algebraic sets. Indiana Univ. Math. J. 1993, 42, 969-984. [CrossRef]

34. Vasilescu, F.H. Spectral measures and moment problems. In Spectral Analysis and its Applications; Ion Colojoară Anniversary Volume; Theta: Bucharest, Romania, 2003; pp. 173-215.

35. Norris, D.T. Optimal Solutions to the $\mathrm{L}_{\infty}$ Moment Problem with Lattice Bounds. Ph.D. Thesis, University of Colorado, Denver, CO, USA, 2003. 
36. Olteanu, O. Mazur-Orlicz theorem in concrete spaces and inverse problems related to the moment problem. UPB Sci. Bull. Ser. A 2017, 79, 151-162.

37. Niculescu, C.P.; Olteanu, O. A Note on the Isotonic Vector-Valued Convex Functions. Preprint. arXiv 2020, arXiv:2005.01088v. Available online: https://arxiv.org/abs/2005.01088 (accessed on 22 September 2020).

38. Niculescu, C.P.; Olteanu, O. From the Hahn-Banach extension theorem to the isotonicity of convex functions and the majorization theory. RACSAM 2020, 114, 171, 1-19. [CrossRef]

39. Montiel, López, P.; Galán, Ruiz, M. Revisiting the Hahn-Banach theorem and nonlinear infinite programming. J. Math. Anal. Appl. 2017, 455, 1037-1050. [CrossRef]

40. Galán, Ruiz, M. A version of the Lax-Milgram theorem for locally convex spaces. J. Convex Anal. 2009, 16, 993-1002.

41. Simons, S. The asymmetric sandwich theorem. J. Convex Anal. 2013, 20, 107-124.

42. Simons, S. Bootstrapping the Mazur-Orlicz-König theorem and the Hahn-Banach-Lagrange theorem. J. Convex Anal. 2018, 25, 691-699.

(C) 2020 by the author. Licensee MDPI, Basel, Switzerland. This article is an open access article distributed under the terms and conditions of the Creative Commons Attribution (CC BY) license (http://creativecommons.org/licenses/by/4.0/). 